\title{
Investigation of Optical Knife Edge Sensor for Low-cost, Large- range and Dual-axis Nanopositioning Stages
}

\author{
ChaBum Lee, ${ }^{1}$ Seongkyul Jeon, ${ }^{1}$ Christopher K. Stepanick, ${ }^{1}$ Abolfazl A. Zolfaghari ${ }^{1}$, and \\ Joshua A. Tarbutton ${ }^{2}$ \\ ${ }^{1}$ Department of Mechanical Engineering, Tennessee Technological University \\ 1 William L Jones Dr., Cookeville, TN, 38505, USA \\ ${ }^{2}$ Department of Mechanical Engineering and Engineering Science, University of \\ North Carolina, 9201 University City Blvd, Charlotte, NC 28223, USA
}

\begin{abstract}
We analyzed the parameter effects of an optical knife-edge sensor (OKES) and the measurement uncertainty to achieve high linearity, long range, and high accuracy for nanopositioning stage applications. The OKES utilizes interference fringes produced from diffraction across the knife-edge. The total field at the detector was calculated from superposition of the incident field and the diffracted field at the knifeedge, and the edge diffraction effects on the sensor design parameters (distance between the knife-edge and detector, wavelength and beam diameter) were investigated by using a design of experiment (DOE), 3-Level $L_{9}$ matrix from the Taguchi Method. Multi-factor experiments were designed to determine the relationship between factors affecting the interferogram and the sensor linearity. It was found that a shorter knife edge-detector distance, shorter wavelength, and larger beam diameter show high signal-tonoise ratio for sensing linearity. The OKES was modeled by using the electromagnetic wave propagation principle, and it was experimentally verified by controlling the positioning of an $X Y$ nanopositioning stage by the OKES. The sensor noise level showed $X 20.1 \mathrm{~nm}$ and $Y 19.4 \mathrm{~nm}$, and the fundamental sensing limits of the OKES were estimated to be $X 0.19 \mathrm{~nm} / \sqrt{H z}$ and $Y 0.23 \mathrm{~nm} / \sqrt{H z}$ for a $\pm 1.0 \mathrm{~mm}$ working range. These results indicate that the OKES can be a good alternative to other precision metrology tools because of its large working range, positioning accuracy, resolution, linearity, and bandwidth, as well as its compact size and low cost.
\end{abstract}

Keywords: Sensor design, Knife edge, Edge diffraction, Nanopositioning, Long-range.

\section{Introduction}

Nanopositioning systems are mechanical motion systems capable of nanometric dimensional precision for accuracy and resolution. Nanopositioning systems are the key to major breakthroughs in a variety of research areas and industrial applications including scanning probe microscopy [1-3], nanometrology [4-7], lithography [8-10], and semiconductor inspection [11]. Achieving large displacement range $(>1 \mathrm{~mm})$ along with nanometer level displacement accuracy $(<50 \mathrm{~nm})$, simultaneously, has been a key challenge in nanopositioning systems due to the physical limitations associated with integrating the driving mechanisms, actuators, and sensors. Currently, many commercially-available nanopositioning stages are restricted to a range of approximately a few hundred micrometers per axis. There is, however, a growing market need for multi-axis nanopositioning systems that can 
provide a millimeter range while maintaining nanometer level dimensional accuracy with a compact integration and low cost.

Most nanopositioning stages rely on flexure linkages to provide linear or rotational motions with no friction or backlash. Their monolithic configuration entirely eliminates friction and backlash, while also providing some additional advantages: nanometric precision and a maintenance free, potentially infinite, lifetime [12-14]. Along with its flexure design, a selection of proper sensors is key to fast and robust positioning control and high resolution measurement.

Many studies on flexure design have been introduced in the past decade [15], but compact and low cost sensors that can be simply integrated with nanopositioning systems have been rarely found. As illustrated in Fig. 1, non-contact sensors such as capacitive-type sensors (CS), laser interferometers, and laser encoders have been typically used due to their ability to perform dynamic motion characterization with a fast, high resolution measurement $[16,17]$. The $\mathrm{CS}$ is the most common because it can be integrated with the stage easily due to its compact size. However, the CS is limited to a working range of tens to hundreds of micrometers. Also, these systems include conductive targets and the attractive force between the target and the sensor probe should be considered for precision applications [18]. While miniature versions using fiber-optic cables have become available recently, their integration within compact nanopositioning systems remains a challenge [19]. Strain gauges and piezoelectric sensors have also been used to measure displacement [20,21]. However, the noise level of strain gauges is too high to detect the displacement with sufficient bandwidth and nanometer resolution, and the piezoelectric sensors do not allow the measurement of low frequency or static displacement.

In general, knife-edges are used for characterizing optical spots as spatial filters and have been implemented in optical beam deflection based on in-plane displacement sensing [22,23]. Optical displacement sensors by means of knife-edges have been recently introduced. In particular, Braunsmann proposed an optical knife edge technique to detect and correct the nonlinear displacement in high speed atomic force microscopy [24]. Karabacak used a knife edge technique to measure sensitive in-plane displacement in nanomechanical beams [25]. Lockerbie proposed a displacement magnification method by using a knife-edge [26]. Strictly speaking, the research above only used knife-edges to partially block the transverse light, and the edge diffraction phenomenon has not been considered. Lee characterized the effect of the parameters of knife-edge diffraction sensing sensitivity based on an edge diffraction theory and showed that edge diffraction at the knife-edge can increase measurement sensitivity [27]. However, their measuring ranges are limited to a few tens of micrometers even though they achieved nanometer resolution because the detector was assumed to be a point. Also, Lee proposed a single axis flexure mechanism with a knife-edge based displacement sensor $[16,17,28]$. The lensed photodetectors were used to increase the measuring range, but it is limited to $500 \mu \mathrm{m}$ and the resolution was $50 \mathrm{~nm}$ because of the lens effect.

In the previous section we outline the physical challenges associated with the displacement sensor to fulfill the requirements of nanopositioning systems: compact, multi-axis, high resolution, millimeter working range, easy system integration, and low cost. Our sensor utilizes optical knife edge (OKE) diffraction and provides non-contact displacement sensing. In the following sections we establish design principles for an optical knife edge sensor (OKES) which provide a millimeter working range and nanometer level accuracy for multi-axis nanopositioning. Furthermore, a mathematically derived theoretical model for an OKES is derived and experimentally verified by using an XY nanopositioning system. Additionally, in this article, the fundamental limits of the OKES are discussed in terms of resolution, linearity, bandwidth, and control effectiveness by using an XY nanopositioning system. 


\section{Theoretical Model}

\section{2-1. Knife-Edge Diffraction}

The measurement method of the OKES is illustrated in Fig. 2. The subscript $i$ and $j$ indicate a coordinate system and a paraxial beam propagating axis separated with a knife edge-detector distance $L, k$ is the wavenumber, and $A$ is the distance between the beam splitter (BS)/Prism and the optical knife-edge (OKE), and $B$ is the distance between the OKE and detector. A laser diode (LD) light is separated by a 50:50 BS. The reflected beam at the BS is incident on the upper side of the OKE mounted on the stage and the transmitted beam at the BS reflects off the prism and is incident to the lower side of the OKE. Each incident beam is partially transmitted and blocked by an OKE position. In electromagnetic wave propagation, however, the OKE acts as a secondary light source, and creates a new wavefront that propagates into the geometric shadow area of the OKE. This is referred to as edge diffraction. Thus the partially transmitted beam and the diffracted beam created from the OKE are superimposed to generate interference fringes or so-called interferograms. The diffracted beam contributes to an increase in the peak power of the $1^{\text {st }}$ fringe by more than $40 \%$ of the transmitted beam power $[29,30]$. The proposed OKES makes use of the optically amplified light signal. This amplification lends itself to producing a high sensor sensitivity. In addition, two signal outputs are differentially amplified and divided by the sum of the two signals. Consequently, the displacement along the traveling direction can be detected simultaneously by using two detectors $\left(D_{1}, D_{2}\right)$.

Assuming that a Gaussian beam with a beam diameter $\alpha$ is incident on the OKE, the total field in front of the OKE is simply the incident field, and the beam propagates along the $z$ axis, the incident field $\vec{E}_{0, j}$ can be defined as:

$$
\vec{E}_{0, j}\left(x_{0, j}, y_{0, j}, z_{j}\right)=E_{j} e^{-\frac{x_{0, j}^{2}+y_{0, j}^{2}}{(\alpha / 2)^{2}}}
$$

where $E_{j}$ is the constant electric field of LD and $\vec{E}_{i, j}$ is the field incident on the $i$ coordinate system along $j$ detector. The last term of Eq (1) is for the aperture of the field. The incident field $\vec{E}_{0, j}$ becomes separated into two fields at the knife edge: the transmitted field, and the diffracted field. The superposition of the two fields creates constructive and destructive interference patterns at the detector. The superimposed wave can be derived by using a Fourier transform (FT), and the total field $\vec{E}_{d, j}$ (sum of transmitted and diffracted field) at $j$ detector can be obtained by applying an inverse FT of the superimposed wave. The total field to be measured along the $\mathrm{z}$ axis can be defined from the inverse FT relation of the incident field as:

$$
\vec{E}_{d, j}\left(x_{d}, y_{d}, z_{i}\right)=\iint_{k-\text { space }} \frac{1}{(2 \pi)^{2}} \iint_{\text {aperture }} \vec{E}_{0, j} \cdot e^{i k \cdot r_{\{s \rightarrow 0\}}} e^{-i k \cdot r_{i 0 \rightarrow d\}}} d y d x d k y d k x .
$$

where $\vec{r}_{i \rightarrow j}$ is a vector from $i$ to $j$ in the coordinate system.

Optical metrology systems can be extremely precise but are fundamentally limited by the surface quality of the optical components. As shown in Fig. 3, a model of a rough knife edge surface is presented and the phase difference between the transmitted and diffracted fields is related to the roughness under the assumption that the variation in heights of the knife edge exhibits a Gaussian distribution. Accordingly, 
the roughness on the knife edge boundary is assumed to have a nonzero mean with a Gaussian probability density function $(p d f)$ [19,20]. Based on this assumption, knife edge surface roughness is modeled in a $p d f$ form as:

$$
\vec{E}_{d, j}^{R}\left(x_{d, j}, y_{d, j}, z_{j}\right)=\iint_{k-s p a c e} \frac{1}{(2 \pi)^{2}} \iint_{a p e r t u r e} \vec{E}_{0, j} \cdot e^{i k \cdot r_{\{s \rightarrow 0\}}} e^{-i k \cdot r_{0 \rightarrow \infty d\}}} \cdot p d f(h) d y d x d k y d k x, p d f(h)=\frac{1}{\sigma \sqrt{2 \pi}} e^{-\frac{h^{2}}{2 \sigma^{2}}} .
$$

Where $R$ represents a rough surface, $h$ is the random height along the $y_{0}$ axis, and $\sigma$ is the standard deviation of the roughness. Here, only the roughness along the $x_{0}$ axis, independent of the $y_{0}$ axis, is considered.

The total power $I_{j}$ induced by the OKE at $D_{j}$ detector can be calculated by multiplying the total field and the conjugated total field at each detector plane and the OKES output can be calculated by Eq. (4) as the OKE is moved along the $y_{0}$ axis.

$$
\begin{gathered}
I_{j}\left(y_{0}\right)=\iint_{\text {detectorsize }} \vec{E}_{0, j}^{R}\left(x_{d}, y_{d}, z_{d}\right) \cdot \vec{E}_{0, j}^{* R}\left(x_{d}, y_{d}, z_{d}\right) d x d y . \\
V_{\text {Out }}=K \times \frac{I_{2}\left(y_{d}\right)-I_{1}\left(y_{d}\right)}{I_{2}\left(y_{d}\right)+I_{1}\left(y_{d}\right)},
\end{gathered}
$$

The differentially amplified output signal can be calculated by Eq. (5), where $K$ is the sensitivity constant of the detector. $\mathrm{K}$ is adjustable by signal amplification, so $V_{\text {out }}$ can be normalized in computation. It uses an output difference of detectors divided by an output sum of detectors, which can effectively eliminate the time-dependent effect of the light source.

\subsection{Parametric Study}

The following design parameters of an OKE sensor were selected: wavelength, beam diameter, and the distance $B$, between the knife edge and PD. The distance between the laser source and knife-edge was not considered because the light source was assumed to follow a Gaussian beam distribution with no divergence. Also, the detector size was initially assumed to be equal to the beam diameter, $\phi 5 \mathrm{~mm}$. The detector size and beam diameter are key parameters to determine the sensor sensitivity and linearity, as well as measuring range as derived in Eq. (4). In parametric design, the detector size is assumed to be the same as the laser beam size to independently analyze the parametric effects on the linearity. Otherwise, the measuring range becomes short when a small detector is used, although a large laser beam is incident on the knife-edge because there is a complex interaction between the detector size and beam diameter. The design of experiment (DOE) $L_{9}$ matrix was used to obtain high sensor linearity at a given measuring range. In this study, the Taguchi method that is commonly employed to search optical parameters to meet the objective function, linearity, was used [31-34]. The effects of interaction between parameters were not considered in this parametric design.

The sensor linearity was evaluated within 80 percent of the maximum detectable size (beam diameter) The DOE matrix used the following design parameters: $B(10 / 20 / 50 \mathrm{~mm}), \lambda(405 / 650 / 830 \mathrm{~nm})$ and $\alpha$ $(0.5 / 1.0 / 5.0 \mathrm{~mm})$. As shown in Fig. 4, the highest signal-to-noise ( $\mathrm{SN})$ ratio for high sensor linearity was found at the following parametric conditions: $B(10 \mathrm{~mm}), \lambda(405 \mathrm{~nm})$ and $\alpha(5.0 \mathrm{~mm})$. Under the optimized set of parameters, the normalized sensor output was calculated from Eq. 4 at detector sizes 
ranging from $0.1 \mathrm{~mm}$ to $5.0 \mathrm{~mm}$ as shown in Fig. 5. Those nonlinearities were estimated in the range of 80 percent of each detecting full scale, respectively. The high nonlinearity, which was calculated as a sum of residual error in least-squares fitting of a linear fitted curve, was found in the case of a small detector size. Figure 6 showed that the nonlinearity becomes $0.3 \%$ in the case of a detector size of $1.0 \mathrm{~mm}$ and can be further reduced to $0.01 \%$ in the case of a $5.0 \mathrm{~mm}$ detector size. It was thought that the averaging effect of the total field over the large detector size offers high linearity.

\section{Experimental Verification}

\section{3-1. Experiment setup}

To verify the proposed mathematical model of the OKES, a flexure stage was constructed to investigate the detector size effect on the sensor linearity. As shown in Fig. 7, the knife edge was placed at the center of the stage aligned without offset distance along the driving axis, a voice coil motor (VCM) was used as an actuator, and a laser diode module (diameter $\phi 5.0 \mathrm{~mm}$ ) equipped with collimation lens was used. The knife-edge used in this experiment was a double edge razor blade (Feather Inc.) with roughness $\sigma \sim 0.4$ $\mu \mathrm{m}$ [26]. This roughness was thought to be sufficiently smooth to create an interferogram. The CS (Lion Precision Inc. measuring range $500 \mu \mathrm{m}, 10 \mathrm{~nm}$ resolution) was used as a reference sensor to measure the displacement and control the positioning of the stage. Two PDs were placed behind the knife edge with a knife edge-detector distance of $10 \mathrm{~mm}$. In this study, two pairs of PDs with different detector sizes $\left(0.1 \times 0.1 \mathrm{~mm}^{2}\right.$ and $\left.3.0 \times 3.0 \mathrm{~mm}^{2}\right)$ were used to test the detector size's effect on the sensor performance.

The OKE sensor output was compared with the analytical results (Fig. 5) and the displacement measured by the CS. The output of the signal conditioning circuit of the OKES was set to $\pm 10 \mathrm{~V}$ due to the acquisition limit of NI LabView software. To illustrate good agreement between theory and the experimental results more clearly, the scale of the OKES output was adjusted. In the case of detector sizes of $0.1 \times 0.1 \mathrm{~mm}^{2}$, the OKE sensor outputs in the forward and backward directions showed good agreement with theoretical estimation results as shown in Fig. 8. It showed a linear range of $60 \mu \mathrm{m}$ with a nonlinearity of $0.7 \%$. Sensor nonlinearity was calculated as an error in a least-squares fitting of a linear fitted curve [30]. The sensor accuracy under this sensor configuration was estimated to be $\pm 0.21 \mu \mathrm{m}$ in the full working range based on the sensor nonlinearity. Similarly, the OKES outputs in the forward and backward directions showed high linearity (nonlinearity $0.6 \%$ ) within a $500 \mu \mathrm{m}$ range (CS measuring limit) using detector sizes of $3.0 \times 3.0 \mathrm{~mm}^{2}$, as shown in Fig. 9. The sensor accuracy was estimated to be $\pm 1.5 \mu \mathrm{m}$ in the full working range. It was thought that this nonlinearity is due to mathematical operations (Eq. (5)) and sensitivity variation of PDs in electronics and electronics signal noise.

\section{3-2. XY System}

The $X Y$ nanopositioning system, $\left(150 \times 150 \mathrm{~mm}^{2}, 20 \mathrm{~mm}\right.$ thickness $)$ based on a parallel kinematic and monolithic configuration, [3] was designed to yield a compact layout as shown in Fig. 10 and provide more than a $\pm 1.0 \mathrm{~mm}$ range. It was fabricated by using a stereolithography process $[17,28]$. From the open-loop testing, the $X Y$ nanopositioning system was characterized: natural frequency $(55.6 \mathrm{~Hz} / 52.6 \mathrm{~Hz})$ and damping ratio (0.14/0.09) along the $X$ and $Y$ axes, respectively. Two OKESs were embedded into the stage and measure its $X Y$ displacement to control the positioning of the stage in real time. The LD $(5 \mathrm{~mW}$, $\lambda 650 \mathrm{~nm}, \alpha 5.0 \mathrm{~mm}$ ) light is 50:50 separated at the BS and travels to each OKES with surface roughness $\sim 0.1 \mu \mathrm{m}$. The VCMs were used as actuators. Four photodetectors $\left(\mathrm{PD}, 3 \times 3 \mathrm{~mm}^{2}\right.$ ) were installed at the 
center of the stage to receive the transmitted and diffracted light behind the OKE with a distance $B=10$ $\mathrm{mm}$, and the PD electronics were set to a bandwidth of $10 \mathrm{kHz}$.

The theoretical sensor output calculated in Eq. (4) shows a sensitivity of $0.667 \times 10^{-3} \mathrm{~V} / \mu \mathrm{m}$ and high linearity (nonlinearity $0.0018 \%$ ) within a $\pm 1.0 \mathrm{~mm}$ range as shown in Fig. $\mathbf{1 1}$. The XY nanopositioning system was proportional-integral-differential (PID) controlled with the OKES and a CS was used for comparison and calibration. The OKES showed a sensitivity of $8.99 \mathrm{mV} / \mu \mathrm{m}$ and $9.02 \mathrm{mV} / \mu \mathrm{m}$ along the $X$ and $Y$ axes, respectively within a $\pm 500 \mu \mathrm{m}$ (CS max. measurement range) with a nonlinearity of $0.60 \%$ and $0.73 \%$ along the $X$ and $Y$ axes, respectively.

A $300 \mu \mathrm{m}$ step test of the $X Y$ stage was performed as shown in Fig. 12. The OKES and CS results showed good agreement and the steady-state positioning root-mean-square (rms) noise, which is also representative of the resolution, was $21.5 \mathrm{~nm}$ and $19.3 \mathrm{~nm}$ along $X$ and $Y$ axes, respectively. We think that the discrepancy of the $X$ and $Y$ step responses results from the additive manufacturing (AM) fabrication tolerances (flexure width, thickness and surface roughness), anisotropy in the material properties, or different VCM characteristics, including the current amplifiers. As a result, the fundamental sensing limit of the OKES in the present experiment setup can be determined by the photonic shot noise $\left(N_{S}\right)$ in Eq. (6) [24,25]:

$$
N_{s}=\Delta R / \sqrt{f}
$$

where, $\Delta f$ and $\Delta R$ are the measurement bandwidth and resolution, respectively. The photonic shot noise indicates the variation of the sensing signal as a function of its resolution and measurement bandwidth. Thus, the fundamental sensing limit of the OKES was estimated: $X 0.19 \mathrm{~nm} / \sqrt{H z}$ and $Y 0.23 \mathrm{~nm} / \sqrt{H z}$ for a $\pm 1.0 \mathrm{~mm}$ full range. This noise can be reduced by using a sufficiently bright light source with a large beam diameter relative to the PD [6].

\section{3-3 Uncertainty}

The measurement uncertainty of the proposed displacement sensor can be calculated as a sum of all uncertainties, as summarized in Table 1. Under the condition that the distance $B$ is long enough and the beam diameter is relatively larger than the detector size; beam diameter $\phi 5.0 \mathrm{~mm}, \mathrm{PD}, 3 \times 3 \mathrm{~mm}^{2}$, distance B $10 \mathrm{~mm}$, and PD electronics bandwidth $10 \mathrm{kHz}$, the uncertainty was calculated with respect to the parametric errors in percentage (wavelength, distance (B), beam diameter) as shown in Fig. 14. The displacement error was calculated by multiplying $V_{\text {out }}$ (Eq. 4) by an amplification gain $(\times 200)$, which produces a final output range of $\pm 10 \mathrm{~V}$. It can be seen that the parameters, distance and wavelength are significant uncertainty sources. The standard deviation of each uncertainty source is summarized in Table 1. The combined standard uncertainty, known as the root sum of the squares, was estimated to be 372.3 $\mathrm{nm}$ in the full range under current experimental conditions. It was found that the linearity error was the biggest uncertainty source. This indicates that an intensity-stabilized laser system is required in this system, and it was thought that a proper look-up table can solve this issue. The uncertainty sources of the laser parallelism could be one of most important error sources in the sensor. In the theoretical approach, it is assumed that the Gaussian laser beam propagates along the traveling axis with no beam divergence, and the laser beam is incident to the detector. In this work, off-axis optics problems were not included because the theoretical OKES model cannot be applied to off-axis optics problems.

Table 1. Uncertainty estimation and calculation. 


\begin{tabular}{|l|l|l|r|}
\hline Error sources & \multicolumn{1}{|c|}{ Errors } & \multicolumn{1}{|c|}{ Mathematical form } & Uncertainty [nm] \\
\hline \multirow{5}{*}{ Optical sources } & Wavelength variation $\sim 20 \mathrm{~nm}$ & See Figure 14. & 30.5 \\
\cline { 2 - 4 } & Laser intensity stability & $\begin{array}{l}\text { Minimized by using a } \\
\text { differential amplifier. }\end{array}$ & 21.5 \\
\cline { 2 - 4 } & Beam divergence $0.6 \mathrm{mrad}$ & See Figure 11. & 0.0 \\
\hline \multirow{2}{*}{$\begin{array}{l}\text { Environmental } \\
\text { sources [5] }\end{array}$} & Air refractive index $\sim 0.0001 \% /{ }^{\circ} \mathrm{C}$ & $\begin{array}{l}\text { Too small to be } \\
\text { considered. }\end{array}$ & $\sim 0.0$ \\
\hline \multirow{2}{*}{$\begin{array}{l}\text { Mechanical } \\
\text { sources }\end{array}$} & $\begin{array}{l}\text { Distance } \Delta \mathrm{B} \text { due to thermal } \\
\text { expansion } \alpha_{\mathrm{T}} \text { of the polymer }\end{array}$ & $\begin{array}{l}\mathrm{B} \times \boldsymbol{\alpha}_{\mathrm{T}}(73.8 \mu \mathrm{m} /(\mathrm{mK})) \\
\times 6^{\circ} \mathrm{C}(\operatorname{Ref}[27])=22.1 \\
\mu \mathrm{m} . \text { See } \text { Figure 14. }\end{array}$ & $\sim 0.0$ \\
\cline { 2 - 4 } & Laser parallelism & Not included. & 0.0 \\
\cline { 2 - 4 } & Edge surface roughness $\sigma 0.4 \mu \mathrm{m}$ & Smooth, Ref. [27] & 0.1 \\
\hline \multirow{2}{*}{ Electric sources } & Reading error $(20 \mathrm{bit})$ & $2.0 \mu \mathrm{V} \div 2 \div 8.99 \mathrm{mV} / \mu \mathrm{m}$ & 370.4 \\
\cline { 2 - 4 } & Linearity error in full range & $3.33 \mathrm{mV} \div 8.99 \mathrm{mV} / \mu \mathrm{m}$ & 5.2 \\
\cline { 2 - 4 } & Drift & Ref. [27] & 372.3 \\
\hline \multirow{2}{*}{ Total } & Uncertainty $\sqrt{\sum U_{i}^{2}}$ where, $U_{i}$ is an i-th uncertainty. & \\
\hline
\end{tabular}

\section{Conclusion}

The OKES design principle was investigated by analyzing the OKES parameter effects to achieve high linearity, a long range and high accuracy for nanopositioning stage applications. The OKES mathematical model was developed and its characteristics were studied in terms of working range, positioning accuracy, resolution, linearity, and bandwidth with the $X Y$ nanopositioning system. From the theoretical approach, we found that this sensing technique is advantageous in achieving high linearity over a millimeter-level range and high bandwidth when compared to the CS, but the OKES design parameters have a significant influence on the sensor performance and uncertainty. Thus, the sensor design parameters have to be optimized for a specific objective such as measuring range. In the current experimental setup, the results showed that the OKES is capable of a large working range $(>1 \mathrm{~mm})$ with nanometric resolution $(<50 \mathrm{~nm})$ for single-axis or multi-axis operation. The OKES is compact and has a low cost. This sensor can be good alternative to CSs, optical encoders, and possibly even a better sensor in nanopositioning applications such as high resolution microscopy, surface profiling, or coordinate measuring. For future work, we will investigate the measurement uncertainty estimation of the OKES by properly characterizing environmental effects, the OKE topography, and optical alignment tolerance.

\section{Acknowledgements}

The research was supported by NSF (Award Number: CMMI 1463502) through Tennessee Technological University. Similarly, this work was supported by the Center for Manufacturing Research, and the authors thank Mr. David Westbury and Dr. David Rocheleau at the University of South Carolina for their stereolithography work.

\section{References}


[1] G. Dai, F. Pohlenz, H-U. Danzebrink, M. Xu, K. Hasche and G. Wilkening, Metrological large range scanning probe microscope, Rev. Sci. Instrum. 75(4), 962-969 (2004).

[2] J. A. Kramar, Nanometre resolution metrolology with the molecular measuring machine, Meas. Sci. Tech. 16, 2121 (2005).

[3] A. Ptak, M. Kappl and H. J. Butt, Modified atomic force microscope for high-rate dynamic force spectroscopy, Appl. Phys. Lett. 88(26), 263109 (2006).

[4] C. Lee, G-H. Kim and S-K. Lee, Design and construction of a single unit multi-functional optical encoder for a six-degree-of-freedom motion error measurement in an ultraprecision linear stage, Meas. Sci. Techno. 22, 105901 (2011).

[5] C. Lee, Gyu-Ha Kim and S-K. Lee, Uncertainty Investigation of Grating Interferometry in Six-degree-offreedom Motion Error Measurements, Int. J. Precis. Eng. Manuf. 13(9), 1509-1515 (2012).

[6] W. Gao, Precision nanometrology: Sensors and measuring systems for nanomanufacturing, Springer (2010).

[7] H-L. Hsieh, J-C. Chen, G. Lerondel and J-Y. Lee, Two-dimensional displacement measurement by quasicommon-optical-path heterodyne grating interferometer, Opt. Express, 19(10), 9770-9782 (2011)

[8] P. C. Paul, A. W. Knoll, F. Holzer, M. Despont and U. Duerig, Rapid turnaround scanning probe nanolithography, Nanotechnology 22(27), 275306 (2011).

[9] A. Weckenmann and J. Hoffmann, Long range 3D scanning tunneling microscopy, CIRP Annals Manuf. Technol. 56(1), 535-528 (2007).

[10] K. Salaita, Y. Wang and C. A. Mirkin, Applications of dip-pen nanolithography, Nat. Nanotechnol. 2(3), 145-155 (2007).

[11] H. Liu, B. Lu, Y. Ding, Y. Tang and D. Li, A motor-piezo actuator for nano-scale positioning based on dual servo loop and nonlinearity compensation, J. Micromech. Microeng. 13, 295 (2003).

[12] Y. K. Yong, S. O. R. Moheimani, N. J. Kenton and K. K. Leang, Invited Review Article: High-speed flexure-guided nanopositioning: Mechanical design and control issues Rev. Sci. Instrum. 83, 121101 (2012).

[13] G. Parmar, K. Barton and S. Awtar, Large dynamic range nanopositioning using iterative learning control, Prec. Eng. 38(1), 48-56 (2014).

[14] S. T. Smith, V. G. Badami, J. S. Dale and Y. Xu, Elliptical flexure hinges, Rev. Sci. Instrum. 68(3), 14741483 (1997).

[15] S. T. Smith, Flexures:Elements of Elastic Mechanisms (Taylor \& Francis, Londong, England, (2002).

[16] C. Lee, T. Corbett, J. A. Tarbutton, Long-term positioning effectiveness of additive manufacturedmonolithic double compound notch type flexure stage, Proceeding of American Society for Precision Engineering 2015 Topical Meeting, April 26-29 (2015).

[17] C. Lee, J. A. Tarbutton and S-K. Lee, Cross-coupling effect of large range XY nanopositioning stage fabricated by stereolithography process, Precision Engineering, Vol. 46, pp. 81-87, (2016).

[18] J. A. Arnaud, W. M. Hubbard, G. D. Mandeville, B. de la Clavière, E. A. Franke, and J. M. Franke, Technique for Fast Measurement of Gaussian Laser Beam Parameters, Appl. Opt. 10(12), 2775-2776 (1971).

[19] L. Perret, L. Chassagne, S. Topcu, P. Ruaux, B. Cagneau, Y. Alayli, Fiber optics sensor for sub-nanometric displacement and wide bandwidth systems, Sens. Actuators A, 165, 189-193 (2011).

[20] G. Schitter, Improving the Speed of AFM by Mechatronic Design and Modern Control Methods, Tech. Mess. 76(5), 266-273 (2009).

[21] A. J. Fleming and K. L. Leang, Integrated strain and force feedback for high-performance control of piezoelectric actuators, Sens. Actuators A 161, 256-265 (2010).

[22] A. H. Firester, M. E. Heller, and P. Sheng, Knife-edge scanning measurements of subwavelength focused light beam, Appl. Opt. Vol. 16, Iss. 7, pp. 1971-1974 (1977). 
[23] J. W. Wagner, Optical detection of ultrasonic, Phys. Acous., Vol. 19, pp. 201-266 (1990).

[24] C. Braunsmann, V. Prucker, and T. E. Schäffer, Optical-knife-edge displacement sensor for high-speed atomic force microscopy, Appl. Phys. Lett. 104, 103101 (2014).

[25] D. Karabacak, T. Kouh, C. C. Huang, and K. L. Ekinci, Optical knife-edge technique for nanomechanical displacement detection, Appl. Phys. Lett. 88, 193122 (2006).

[26] N. A. Lockerbie, A displacement-doubling prism, Nucl. Instrum. Meth. A., Vol. 741, pp. 192-195, (2014).

[27] C. Lee, S-K. Lee and J. A. Tarbutton, Novel design and sensitivity analysis of displacement measurement system utilizing knife edge diffraction for nanopositioning stages. Rev. Sci. Instrum. 85, 095113 (2014).

[28] C. Lee, S-K. Lee and J. A. Tarbutton, Positioning control effectiveness of optical knife edge displacement sensor-embedded monolithic precision stage, Sens. Actuators A. Vol. 233, pp. 390-296, (2015).

[29] C. L. Giovaneli, An analysis of simplified solutions for multiple knife-edge diffraction, IEEE Trans. Antennas Propag. 32(3), 297-301 (1984).

[30] B. A. David and G. S. Brown, Diffraction by a randomly rough knife edge, IEEE Trans. Antennas Propag. 50(12), 1769-1788 (2012).

[31] C. Lee, K. Hane, W. Kim and S-K. Lee, Design of retrodiffraction gratings for polarization-insensitive and polarization-sensitive characteristics by using the Taguchi method, Appl. Opt. 47(18), 3246-3253, (2008).

[32] Y. Wu and A.Wu, Taguchi Methods for Robust Design (American Society of Mechanical Engineers, 2000).

[33] K.-C. Fan, W. Wang, and H.-S. Chiou, Fabrication optimization of a micro-spherical fiber probe with the Taguchi method,J. Micromech. Microeng. 18, 015011 (2008).

[34] C.-K. Huang, H.-H. Nien, S.-K. Changchien, and H.-W. Shieh, Image encryption with chaotic random codes by grey relational grade and Taguchi method, Opt. Commun. 280, 300310 (2007). 


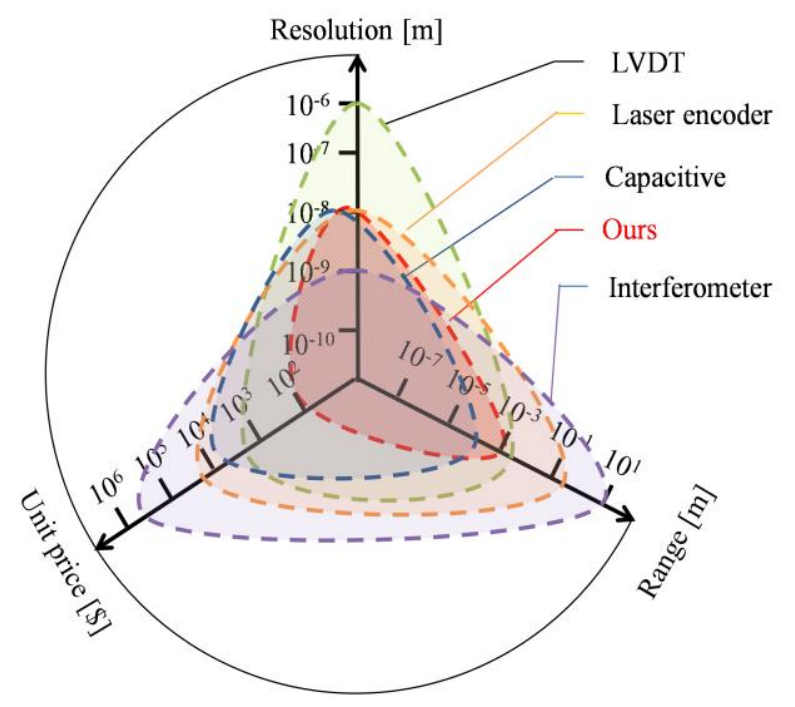

Figure 1. Comparison of various displacement sensors.

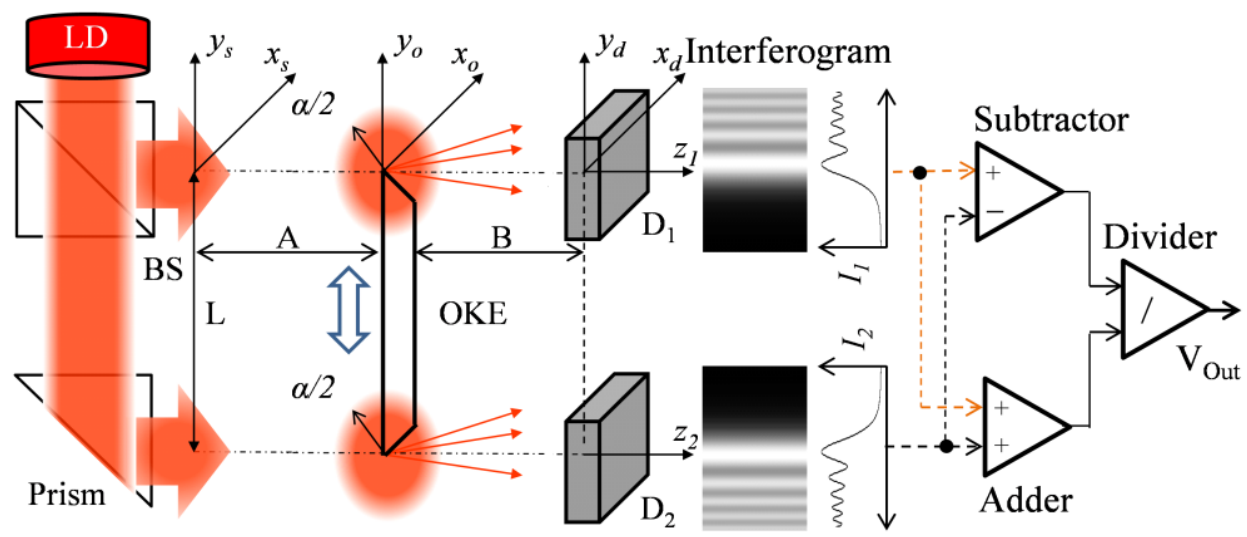

Figure 2. Working principle of the OKES: $x_{i}$ and $y_{i}$ is a coordinate system at $z=z_{j}$ and $D_{j}$ is $j$-th detector at $z=z_{d}$, respectively. 


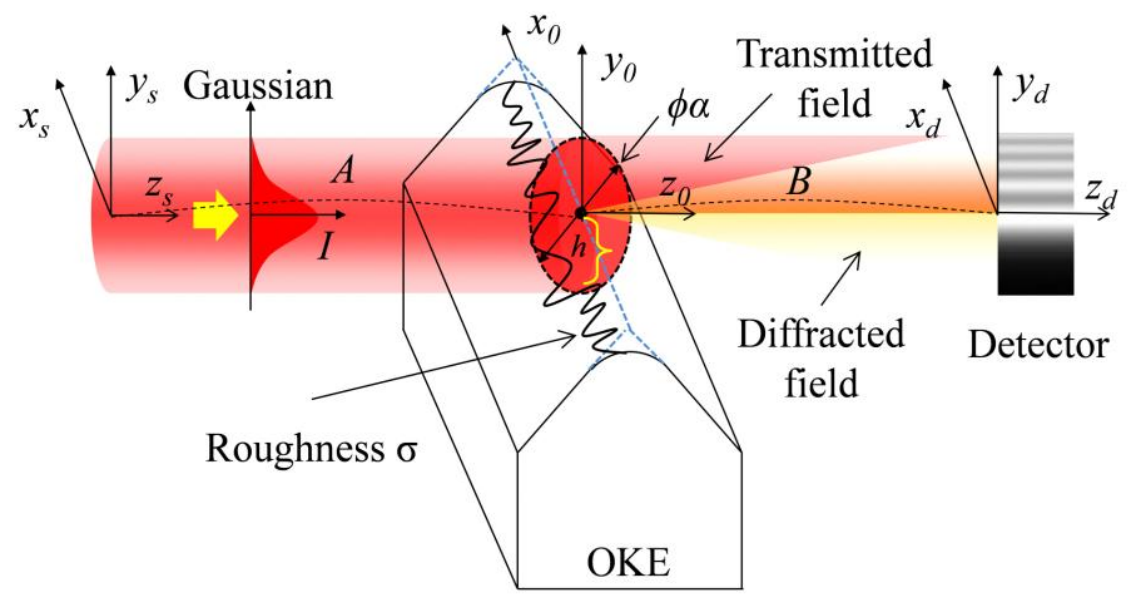

Figure 3. Knife edge diffraction at a rough edge.

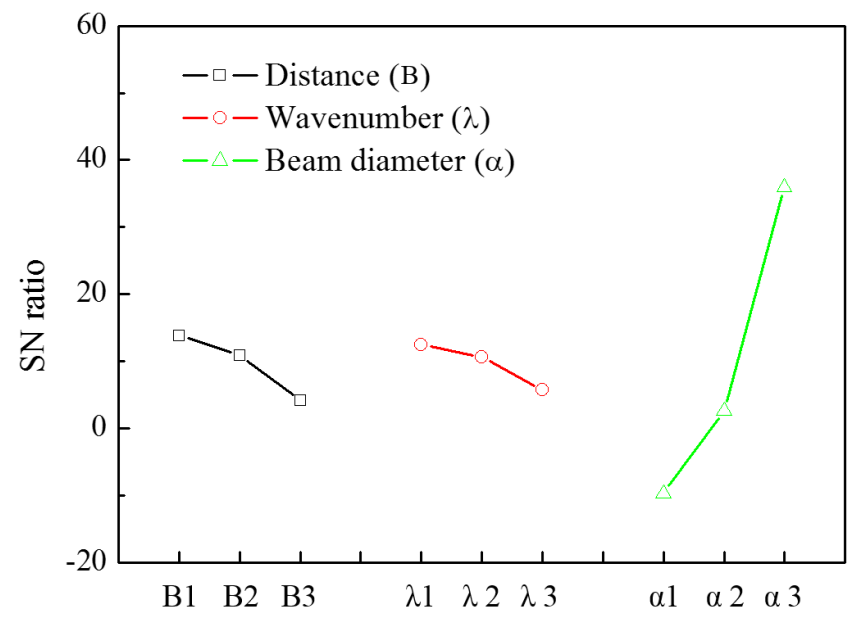

Figure 4. Sensitivity analysis of 3-level variable optimization. 


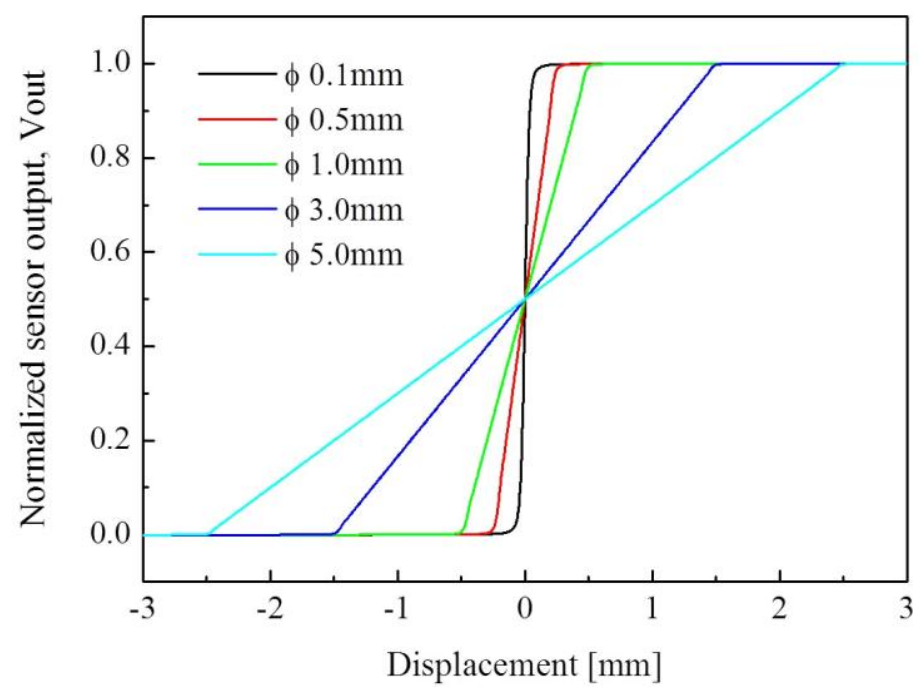

Figure 5. Detector diameter $(\phi)$ effects on estimated sensor output.

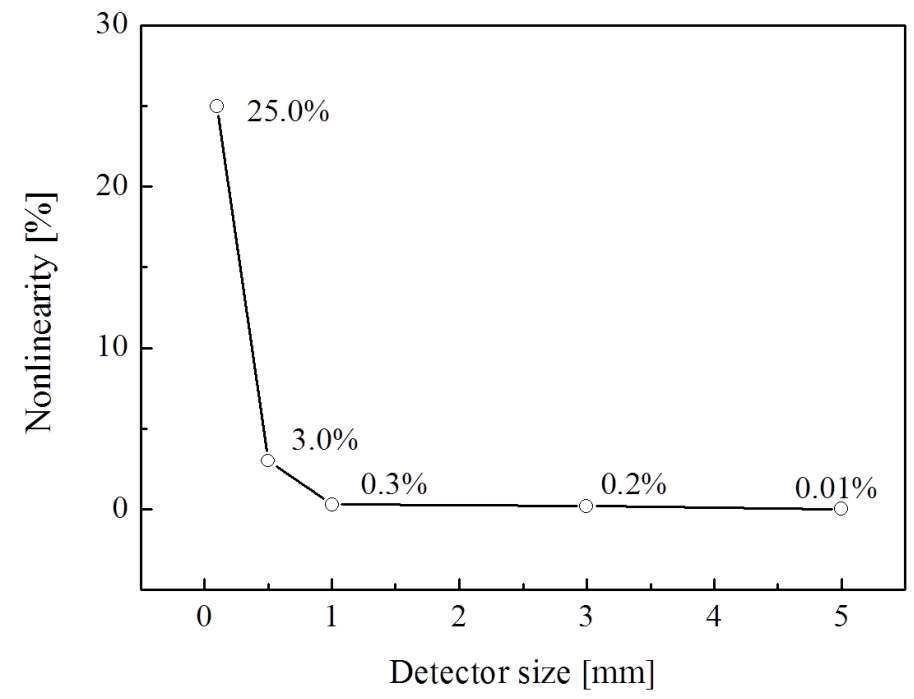

Figure 6. Sensor nonlinearity characteristics with respect to detector size. 


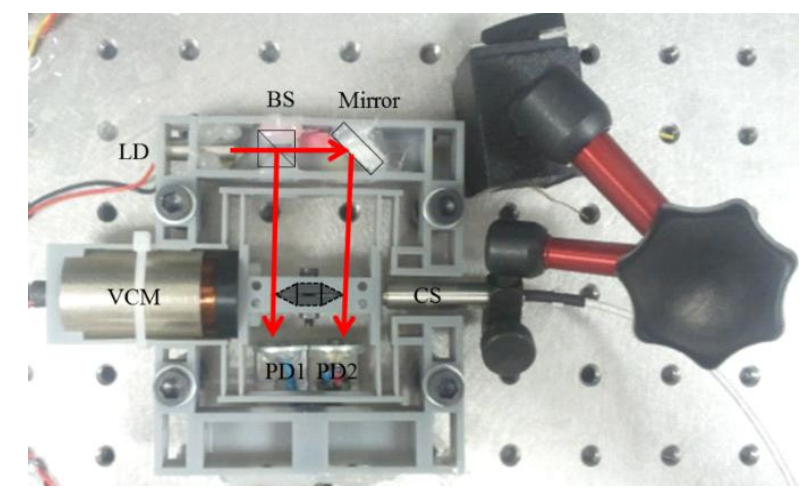

Figure 7. Experiment setup.

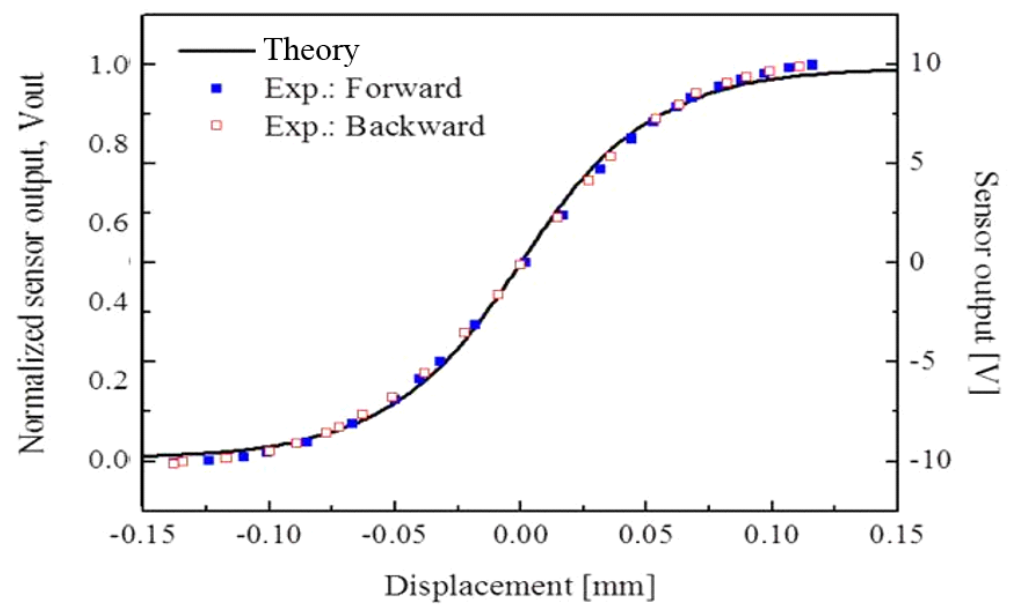

Figure 8. Experiment result: detector size $\left(0.1 \times 0.1 \mathrm{~mm}^{2}\right)$.

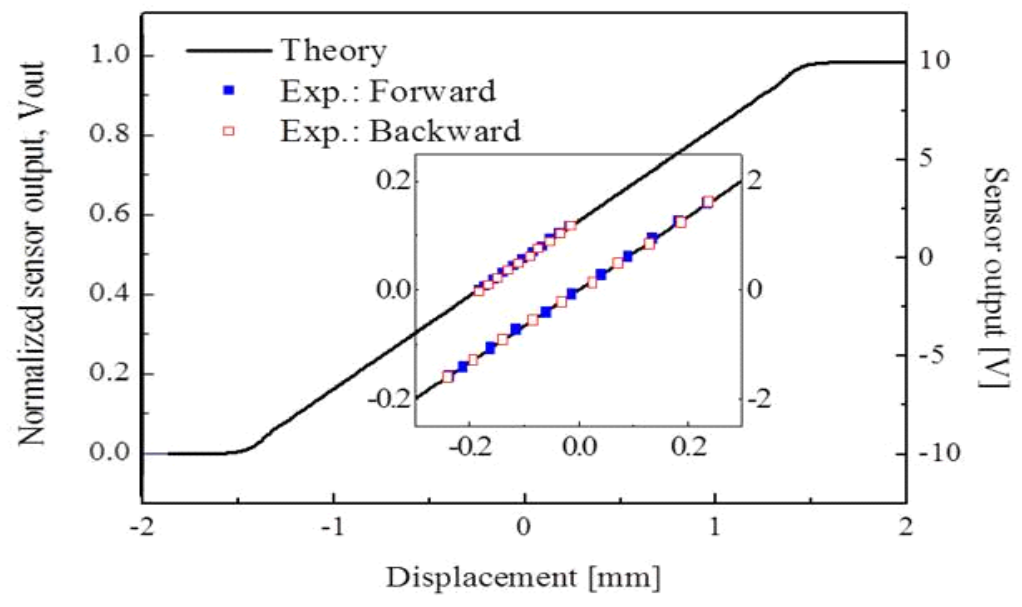

Figure 9. Experiment result: detector size $\left(3.0 \times 3.0 \mathrm{~mm}^{2}\right)$. 


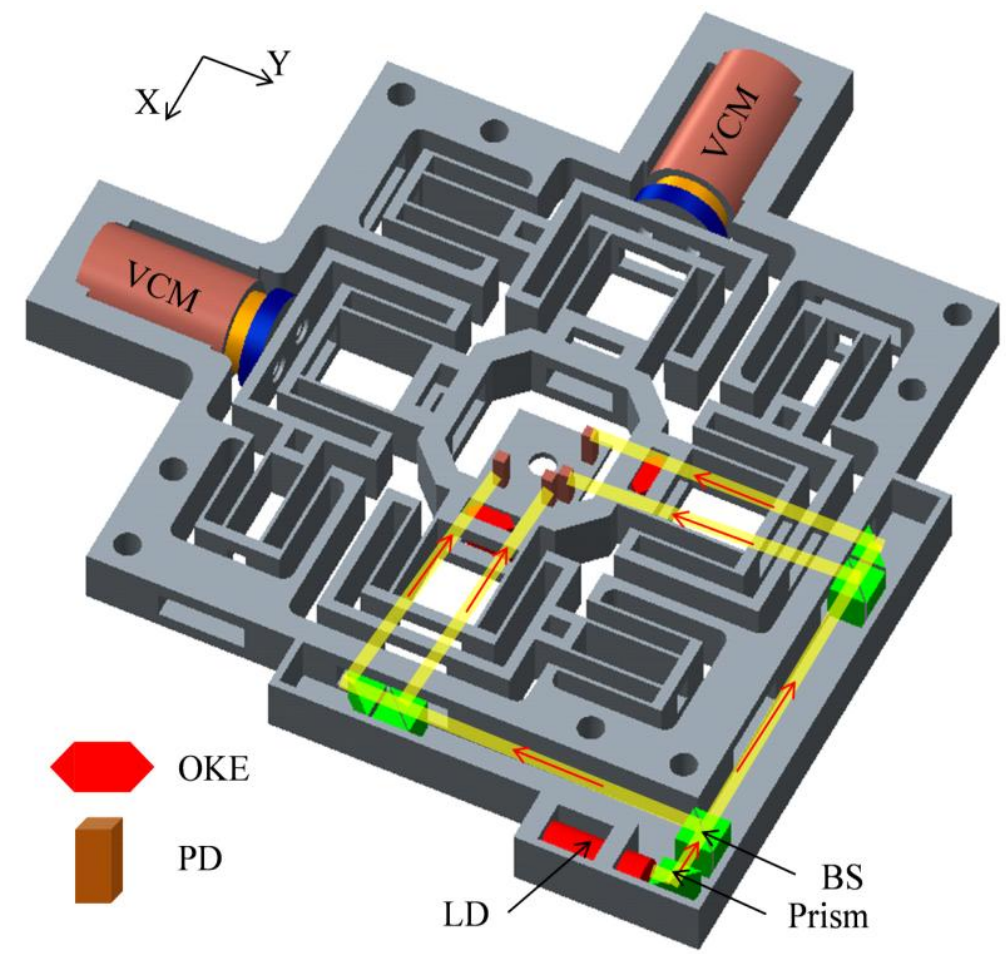

Figure 10. Schematic illustration of the OKES-embedded nanopositioning system: The yellow lines indicate the laser beam path and the red arrows indicate the light propagating direction, respectively. The VCMs were aligned along the moving axis of the stage without offset to mitigate Abbe error, and two OKESes were perpendicularly setup each other. The flexure was designed: $20 \mathrm{~mm}$ long, $15 \mathrm{~mm}$ wide and $1 m m$ thick.

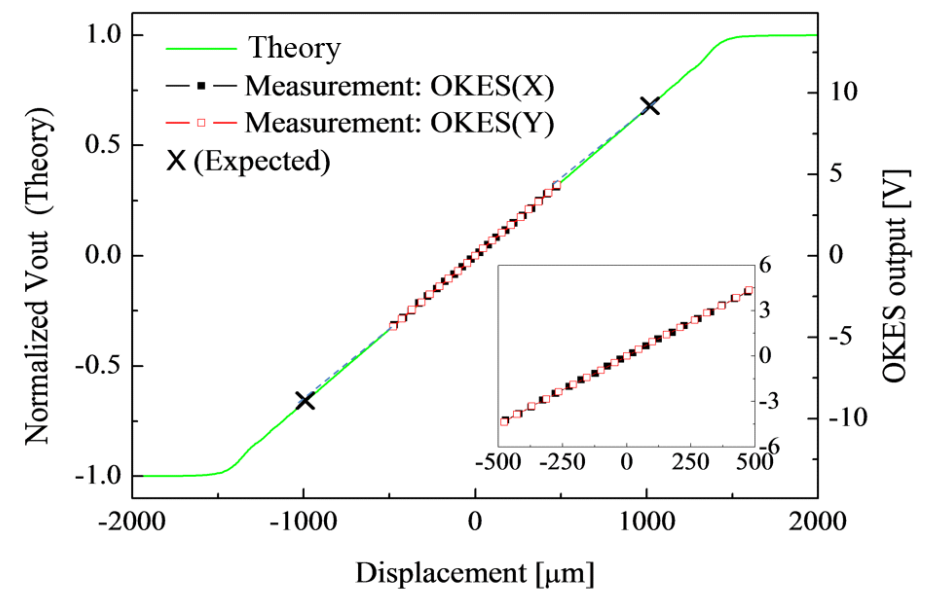

Figure 11. Normalized sensor output calculated with respect to the OKE shift along the $y_{0}$ axis. The theoretical estimation result works for both $\mathrm{X}$ and $\mathrm{Y}$ axes because two OKESes were independently setup each other along the $\mathrm{X}$ and $\mathrm{Y}$ axis. To illustrate good agreement between theory and experiment results more clearly, the scale of the OKES output was adjusted. 

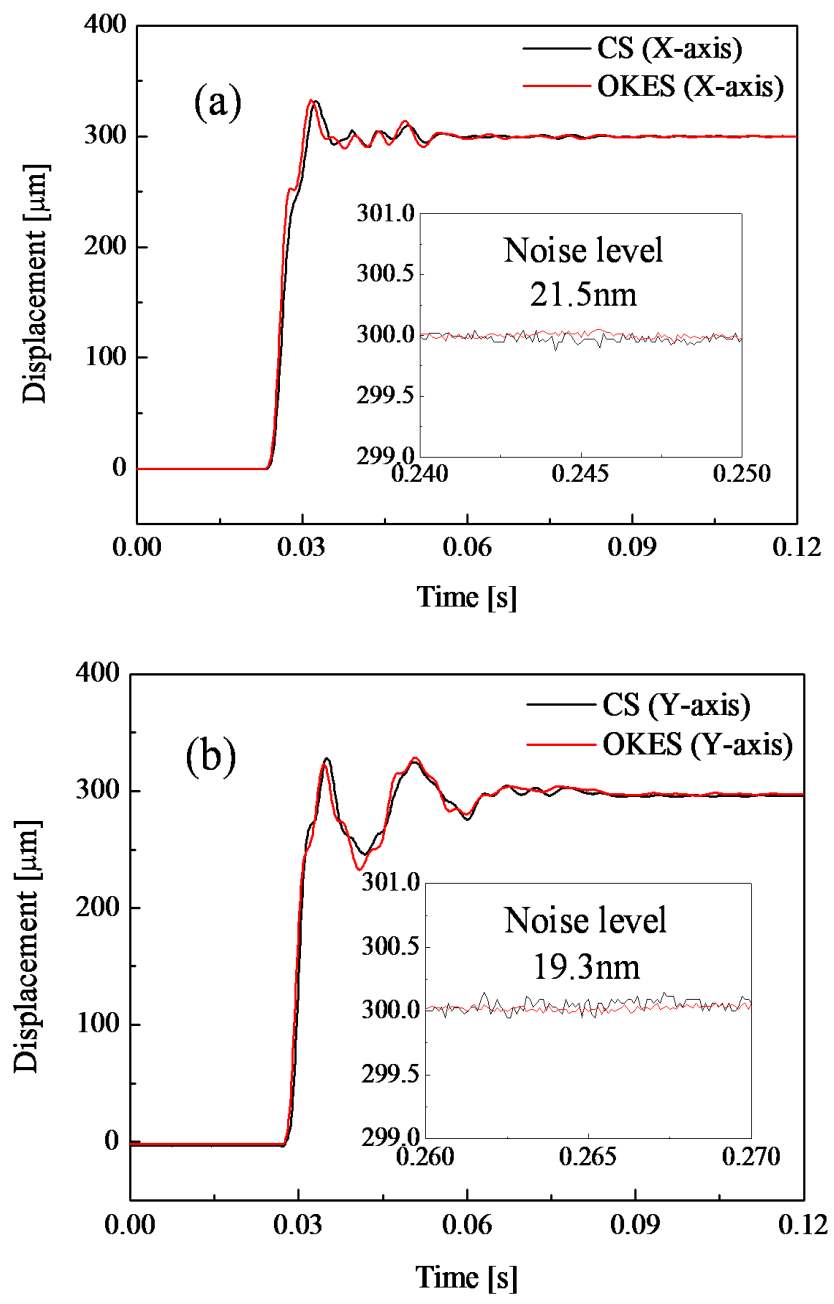

Figure 12. Step response curves of the $X$ (a) and $Y($ b) axes.
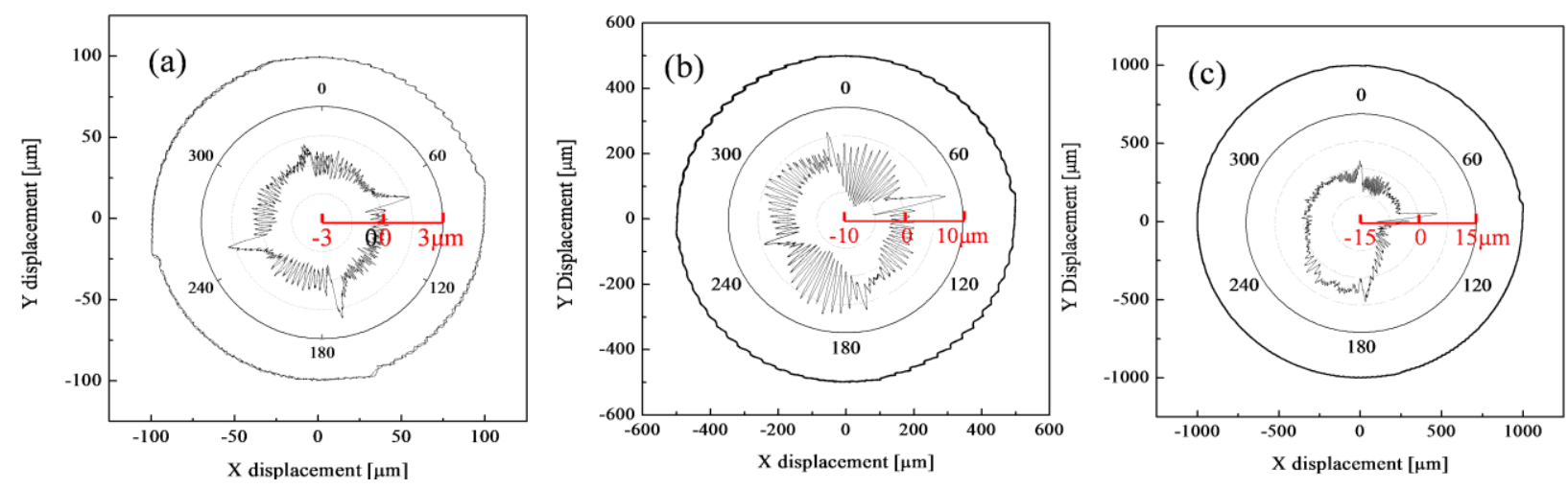

Figure 13. Results of circular motion tracking at $1 \mathrm{~Hz}$ : diameter $0.2 \mathrm{~mm}$ (a), $1.0 \mathrm{~mm}$ (b) and $2.0 \mathrm{~mm}$ (c). The trajectory errors were plotted in a polar coordinate. 


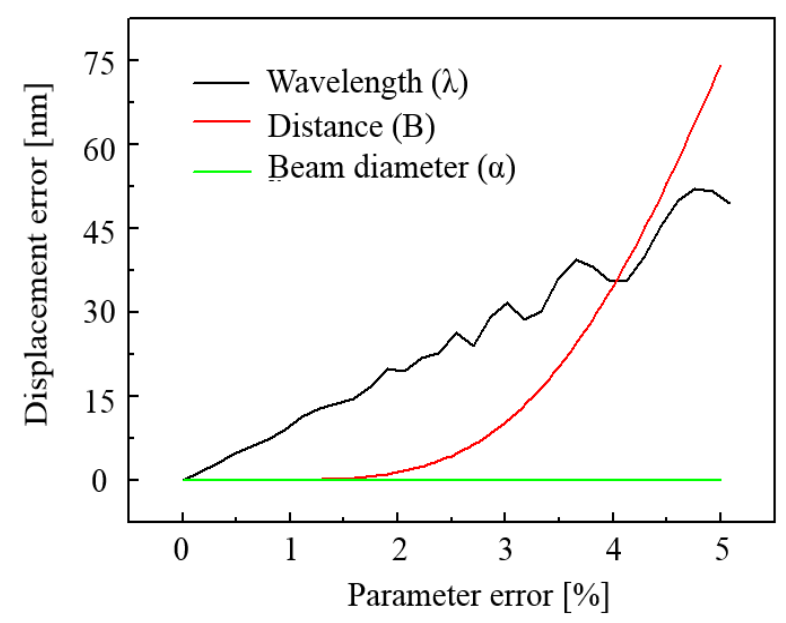

Figure 14. Displacement error curves due to parametric errors. 